\title{
The newest and classic biomarkers of sepsis in HIV-infected adult patients
}

\author{
Tomasz Mikuła, Dagny C. Krankowska, Alicja Wiercińska-Drapało \\ Department of Infectious and Tropical Diseases and Hepatology, Medical University of Warsaw, Hospital for Infectious Diseases, Poland
}

\begin{abstract}
Sepsis is one of the major causes of mortality of patients worldwide, and patients with human immunodeficiency virus (HIV)/acquired immunodeficiency syndrome (AIDS) are at higher risk of developing it. Given the importance of quick diagnosis, the demand for sepsis biomarkers is high. In this article, the authors reviewed the available sepsis biomarkers, and assessed whether the biomarkers were analyzed in patients with HIV/AIDS.

We investigated the available literature on classic inflammatory biomarkers, such as procalcitonin (PCT) and interleukin-6 (IL-6) as well as new biomarkers of sepsis, including soluble form of urokinasetype plasminogen activator receptor (suPAR), proadrenomedullin (proADM), soluble triggering receptor expressed on myeloid cells 1 (sTREM-1), growth arrest-specific 6 (Gas6), and microRNA (miRNA) in immunocompetent patients and patients living with HIV/AIDS. Various biomarkers have a diagnostic value (PCT, sTREM-1), others present a prognostic value (suPAR, Gas6, PSP, HBP), and some biomarkers have both values (IL-6, proADM, sCD14-ST, miRNA). Combining at least two different biomarkers has the best potential to bring high sensitivity and specificity of diagnosis.

To our knowledge, many of discussed novel inflammatory biomarkers, such as presepsin, pancreatic stone protein/regenerating protein (PSP/reg), or heparin-binding protein (HBP), were not yet studied in a population of patients with HIV/AIDS and sepsis.

So far, there is not a one biomarker used as a golden standard in diagnosis of sepsis. Monitoring at least two biomarkers might increase the chance of early detection of sepsis. Further research is needed to find biomarkers diagnosing sepsis in patients with AIDS.
\end{abstract}

HIV AIDS Rev 2021; 20, 4: 257-263

DOI: https://doi.org/10.5114/hivar.2021.111536

Key words: sepsis biomarkers, sepsis, HIV/AIDS, new inflammatory biomarkers.

\section{Introduction}

According to the Third International Consensus Definition for Sepsis and Septic Shock from 2016, sepsis is defined as life-threatening organ dysfunction caused by a dysregulated host response to infection [1]. Dysregulation of immune response is caused by alterations in systemic cytokine levels. In immunocompetent patients, hyper-inflammatory response is then followed by lymphocyte impairment, and

thus leading to immunosuppression. The response in immunocompromised patients seems to be slightly different [2]. Weak and slow response of innate immune system in human immunodeficiency virus (HIV)-infected patients predisposes them to sepsis. Sepsis is one of the major causes of mortality of patients with HIV/acquired immunodeficiency syndrome (AIDS), with an incidence of about 1,000 per 100,000 population [3]. Symptoms of sepsis in immunosuppressed patients can be non-specific, and in patients with

Article history:

Received: 26.01.2021

Received in revised form: 24.03.2021

Accepted: 24.03.2021

Available online: 30.11 .2021
International Journal of HIV-Related Problems

HIV \& AIDS

R e vi e w 
CD4+ cell count $<200$ cells/ $\mu$ l, opportunistic infections (i.e., pulmonary tuberculosis, Pneumocystis jiroveci pneumonia, tuberculous, and cryptococcal meningitis) occur more frequently, making the diagnosis and management of patients with AIDS and sepsis more challenging than of immunocompetent patents.

Biomarkers, as defined in 2001 by the National Institute of Health are "characteristics that can be objectively measured and evaluated as indicators of normal biological processes, pathological processes, or pharmacological responses to a therapeutic intervention" are helpful in everyday clinical practice to diagnose and evaluate effectiveness of treatment [4]. Currently, the most commonly used classical biomarkers of infection are procalcitonin (PCT) and interleukin-6 (IL-6). There are numerous new markers of sepsis that are being investigated, such as soluble form of urokinase-type plasminogen activator receptor (suPAR), proadrenomedullin (proADM), soluble triggering receptor expressed on myeloid cells 1 (sTREM-1), growth arrest-specific protein 6 (Gas6), microRNA (miRNA), presepsin, heparin-binding protein (HBP), and pancreatic stone protein/regenerating protein (PSP/reg). By 2010, at least 178 different biomarkers of sepsis were reported in the literature [5].

In this article, the authors reviewed the available sepsis biomarkers and evaluated whether the biomarkers were analyzed in a group of patients with HIV/AIDS.

\section{Biomarkers of sepsis}

Commonly used classic inflammatory biomarkers are procalcitonin (PCT) and interleukin (IL)-6.

New inflammatory biomarkers include:

- suPAR - soluble form of urokinase-type plasminogen activator receptor,

- proADM - proadrenomedullin,

- sTREM-1 - soluble triggering receptor expressed on myeloid cells-1,

- Gas6 - growth arrest-specific protein 6,

- miRNA - microRNA,

- presepsin,

- PSP - pancreatic stone protein/regenerating protein (PSP/reg),

- HBP - heparin binding protein.

\section{Classic inflammatory biomarkers}

\section{Procalcitonin}

PCT is a peptide precursor of calcitonin produced by thyroid C cells. During bacterial or fungal infection, PCT is also detected in leukocytes, macrophages, and monocytes of intestines, lungs, and liver.

Bacterial toxins and tumor necrosis factor-alpha (TNF- $\alpha$ ) are the strongest stimuli for PCT serum release. The level of PCT increases within 4 hours of initiation of the inflammatory process, and its' half-life is 22-26 hours.
It has been shown that low concentrations of PCT exclude the presence of bacteremia in patients with fever [6]. A meta-analysis reported sensitivity and specificity of PCT in bacteriemia as $76 \%$ and $70 \%$, respectively [7]. Some PCT scores show a correlation with certain pathogens isolated from blood, such as Escherichia coli, Klebsiella pneumonia, Staphylococcus, or Gram-negative rods [8]. According to latest results, PCT is useful for individual assessment of the required duration of antibiotic therapy [9].

Several articles mention PCT as a marker, which increasing level would correlate with increased risk of hospital mortality of critically ill non-immunocompromised patients [10]. Such correlation was not present in a study by Bele et al., who analyzed immunocompromised patients [11].

PCT is considered to be a more accurate biomarker of bacterial infection than C-reactive protein (CRP), but some authors state that PCT alone is not a good prognostic marker of sepsis, as similar levels appear in survivors and nonsurvivors of sepsis [8].

PCT and CRP might be useful markers in differentiating tuberculosis (TB) and community-acquired pneumonia (CAP). According to Kang et al., PCT and CRP are higher in the course of the latter [12].

\section{Procalcitonin in HIV/AIDS patients}

During HIV infection, the PCT level is normal or slightly above upper normal limit. Similar observations were found in patients with HIV infection and Pneumocystis jiroveci pneumonia (PJP) or Toxoplasma gondii infection.

Schleicher et al. analyzed changes in PCT serum concentrations in HIV-infected patients with TB in comparison to HIV-infected patients with pneumococcal pneumonia. Patients with HIV and pneumococcal pneumonia presented with significantly higher levels of PCT and CRP than those with HIV and MTB infection [13].

Similar results were presented by Nyamande et al., who found statistically significant differences in PCT concentrations between HIV-infected patients with bacterial pneumonia, TB, and PJP. The authors suggested using PCT as a marker for differentiating PJP from TB and other bacterial pneumonia in critically ill immunocompromised patients [14].

Patients with tuberculosis were also analyzed by Janssen et al., who in a prospective cohort study with $60 \mathrm{HIV}$-positive patients with tuberculosis found a correlation between higher levels of procalcitonin and mortality [15].

\section{Cytokines}

In sepsis, both pro- and anti-inflammatory cytokines are released. Interactions between different cytokines in a cytokine storm are complex. Many cytokines involved in immune response to infection have been identified as biomarkers of inflammation and potential markers of sepsis. Those biomarkers can be a valuable addition to diagnostics as follow:

Pro-inflammatory cytokines: IL- 1, IL- 6 , TNF- $\alpha$, and tumor necrosis factor-beta (TNF- $\beta$ ) $[16,17]$. 
IL-1 $\beta$, TNF- $\alpha$, and IL-6 are good predictors of 28-day mortality in patients with sepsis. IL- 6 is produced by MAP kinase and NF- $\mathrm{KB}$ and other pathways in the signaling cascade of sepsis. Its' levels rise before CRP and IL-6 itself, and is considered a marker of sepsis. A 10-fold more or higher than baseline value is considered a fairly early indication of sepsis [8].

Anti-inflammatory cytokines: IL-10, IL-4, transforming growth factor- $\beta$ (TGF- $\beta$ ), and IL-13.

A recent mini review by Morrow et al. described interactions between IL-17, IL-27, and IL-33, and showed how these cytokines play their parts in immunological dysfunction during sepsis, revealing their potential as therapeutic targets. IL-27 produced by dendritic cells (DCs), monocytes, and macrophages, is a potent immunosuppressant. In 2012, Wong et al. demonstrated in their study that a concentration of IL-27 > $5 \mathrm{ng} / \mathrm{ml}$ had a good predictive value for diagnosis of critically ill children with a bacterial infection [18]. In another study conducted by the same authors in adults, the biomarker was not as useful as in the children cohort [19].

\section{Cytokines in HIV/AIDS patients}

In HIV-infected patients, IL-6 levels and age are independent risk factors of hospital mortality, irrespective of HIV/AIDS stage of disease. Prognosis of HIV-positive patients with sepsis could be assessed with a concentration level of several cytokines, including IL-6, IL-10, and granulocyte colony-stimulating factor (G-CSF) [20]. For proper evaluation of an outcome of sepsis, the balance between proand anti-inflammatory cytokines i.e., IL-6 : IL-10 ratio is useful, and this ratio could be also helpful for early prognosis of mortality among patients with PJP [21, 22].

\section{New inflammatory biomarkers}

Predicting the development of sepsis in HIV-positive patients is difficult. New biomarkers of inflammation are promising options for early diagnosis of sepsis. Biomarkers of inflammation presented below include those, which in several studies showed a correlation with sepsis and other inflammatory diseases. Some of these biomarkers were analyzed among HIVinfected patients, while others need further investigation.

\section{Soluble form of urokinase-type plasminogen activator receptor (suPAR)}

The urokinase-type plasminogen activator is present on monocytes, macrophages and other cells. During inflammation, it detaches from the cell surface and becomes a soluble form called 'suPAR'. Levels of suPAR are not specific for sepsis; this biomarker is more useful for prognosis of illness rather than for diagnosis of disease $[19,23]$. In a metaanalysis carried out by Ni Wentao et al., sensitivity and specificity for predicting mortality in patients with sepsis were $70 \%$ and $72 \%$, respectively [24]. In a study by Suberviola et al., on the day of admission to ICU, acute physiology and chronic health evaluation II (APACHE II) and SOFA (sepsisrelated organ failure assessment) scores were compared to CRP, PCT, suPAR, and proadrenomedullin (proADM) levels. In presented results, suPAR and proADM levels were better tools than CRP or PCT in prognosing sepsis and in-hospital mortality [25]. Henriquez-Camacho et al. in their study from 2014 observed that diagnostic value of this marker was not superior to other biomarkers, including CRP, PCT, or soluble triggering receptor expressed on myeloid cells-1 (sTREM-1) [26]. However, as demonstrated in a study by Eugen-Olsen et al., suPAR can be used as a predictive marker of mortality in patients with tuberculosis. Other studies showed that suPAR levels correlated with mortality in patients with Streptococcus pneumonia and Staphylococcus aureus bacteriemia [23].

\section{SUPAR in HIV/ IDS patients}

Ever more attention is drawn to the topic of bacterial translocation and immune activation in patients with HIV. SuPAR is considered to be one of the markers of immune activation; it has not been analyzed as a biomarker of sepsis in HIV patients, but as a predictive biomarker of mortality. One of the first reports demonstrating an association between plasminogen activator system and HIV progression was published in 2000. Sidenius et al. showed that suPAR serum concentration correlated with mortality in HIVinfected patients [27].

In a Kirkegaard-Klitbo study with HIV-infected welltreated patients, non-AIDS comorbidity and all-cause mortality (cancer, cardiovascular, kidney, lung, and liver diseases) demonstrated an independent association with elevation of serum suPAR level [28]. It was shown that suPAR appeared to be an independent predictive marker of myocardial infarction in HIV-1-infected patients [29].

\section{Proadrenomedullin (proADM)}

ProADM present vasodilating and bactericidal activity. A combination of sequential organ failure assessment (SOFA) score and serum mid-regional proadrenomedullin (MRproADM) can be used as predictors of 4-weeks mortality, with sensitivity and specificity of $66.7 \%$ and $86.8 \%$, respectively [30]. In earlier works, proADM was considered a biomarker of prognostic value [25]. In a study from 2017, it was shown that proADM together with SOFA score can be used also as part of diagnosis. Spoto et al. analyzed serum PCT and MR-proADM serum concentration, and SOFA and quick SOFA (qSOFA) scores among patients with sepsis or septic shock. Mid-regional proadrenomedullin (MR-proADM) and SOFA presented similar to PCT-positive likelihood ratio in sepsis and septic shock, and those biomarkers can be included in diagnosis and prognosis of sepsis [31]. In another study, a combination of MR-proADM and PCT demonstrated a probability of 0.998 of diagnosing sepsis [32]. This is 
yet another example on how using several markers instead of one presents higher probability of diagnosis of sepsis.

\section{ProADM in HIV/AIDS patients}

Proadrenomedullin, together with other markers (PCT and copeptin) and nasopharyngeal colonization density, were analyzed in 280 patients with HIV and community-acquired pneumonia. Proadrenomedullin, PCT, and copeptin were positively correlated with nasopharyngeal colonization density, with consecutive $p$-values of $<0.0001,0.008$, and 0.01 . Quantitative nasopharyngeal density with $>8,000$ copies/ $\mathrm{ml}$ was an additional criterion for pneumococcal pneumonia [33]. Therefore, one might consider these markers in diagnosis of CAP in HIV-positive patients.

\section{Soluble triggering receptor expressed on myeloid cells-1 (sTREM-1)}

Triggering receptor expressed on myeloid cells-1 (TREM-1) is a cell surface receptor, which number increases in response to severe fungal or bacterial infections. TREM-1 is mainly expressed on dendritic cells, monocytes, neutrophils, or macrophages. Its' soluble variant, soluble triggering receptor expressed on myeloid cells-1 (sTREM-1), is released to urine, cerebrospinal fluid (CSF), or plasma during infection [34]. STREM-1 is also a promising inflammatory biomarker of pneumonia or sepsis-associated acute kidney injury (AKI) $[35,36]$. In 11 studies, with a total of 1,795 patients, sTREM-1 sensitivity and specificity were evaluated as $79 \%$ and $80 \%$, respectively [37]. STREM- 1 and PCT, together with APACHE II and SOFA scores, can be useful methods of diagnosing sepsis [38]. Dolin et al. suggested combining three biomarkers, including sTREM-1, IL-6, and PCT in diagnosis of early-phase sepsis [8].

\section{STREM-1 in HIV/AIDS patients}

A recent publication on the importance of sTREM-1 assessed the ability of inflammatory response of macrophages or Kupffer cells (KC) to HIV or HCV stimulation. Results of this study show that HIV- and HCV-infected patients show higher TREM-1 expression and CD68 receptors positive cells. In addition, TREM-1 induction by HIV causes chronic inflammation in the liver, which may be important in HIV-infected patients' care [39]. We did not find any data about sTREM-1 as a biomarker in patients with HIV and sepsis.

\section{Growth arrest-specific 6 (Gas6)}

Gas6 belongs to plasma vitamin K-dependent proteins. Apart from being elevated in inflammation, Gas6 plasma levels have been shown to correlate with organ dysfunction and disease severity [40, 41]. A recent study by Stalder et al., contrary to previous papers by other authors, demonstrated that plasma level of Gas6 measured at a 24-hour ICU admission, might predict in-hospital mortality in patients with sepsis [42].

\section{Gas6 in HIV/AIDS patients}

There is little clinical data on the significance of Gas6 in HIV-infected patients. It is known that HIV-1 infection induces apoptosis of HIV-1-infected cells, and other viruses are subjected to macrophage efferocytosis, which protects the host from mostly viral infections. In a study by Cua et al., it has been confirmed that Gas6 protein and protein S, can mediate phagocytosis of HIV-1-infected cells, and that efferocytosis not only removes dead cells, but can also contribute to removal of macrophages of infected live cells [43].

\section{MicroRNA (miRNA)}

MicroRNAs are short sequences (20-24 nucleotides) of RNA that regulate gene expression. Even though miRNA represents only $1 \%$ of human genome, it regulates up to $60-90 \%$ of all protein-coding genes, and is essential for physiological and pathophysiological processes. MiRNAs regulate sepsis by targeting TNF and TLR/NF-kB signaling pathways. Levels of miR-25 had a higher diagnostic accuracy for sepsis than CRP and PCT, and miR-25 could also be used for prognosis of sepsis. Serum levels of various miRNAs can be helpful in pathogen identification, including infections with Staphylococcus aureus, Escherichia coli, Listeria monocytogenes, Mycobacterium tuberculosis, Salmonella enterica, Pseudomonas aeruginosa, Leishmania, etc. [44]. Zhang et al. showed that miR-223-3p levels correlated with pneumocystis, and thus could be used as a biomarker of diagnostic value [45].

\section{MiRNA in HIV/AIDS patients}

Cellular miRNAs play an important role in the viral life cycle. The influence of miRNA on HIV replication needs further research, but it is considered to be a promising novel anti-HIV therapeutic [46]. MiRNA-155 is thought to be a biomarker of $\mathrm{T}$ cell activation and immune response after HIV infection [47].

\section{Presepsin (sCD14-ST)}

Presepsin is a 64-amino acids $\mathrm{N}$-terminal fragment subtype of CD14 (sCD14-ST). Since 2004, presepsin is evaluated as a new biomarker of sepsis, with specificity and sensitivity of $83 \%$ and $78 \%$, respectively $[48,49]$. In a large review by Henriquez-Camacho et al. who analyzed inflammatory biomarkers used for diagnosing sepsis, presepsin showed to be one of the main and most accurate biomarkers. In the present review, according to many analysis and metaanalysis, presepsin proved to diagnose sepsis better than PCT [26]. In another study by Tong et al., presepsin again proved to play an important role and predicting value of sepsis in comparison to PCT, CRP, or white blood cells (WBC). It only takes 17 minutes to test presepsin, and fast results make it a very good candidate for a sepsis biomarker [50]. In HIV/AIDS patients, presepsin has been mainly evaluated as one of the biomarkers of bacterial translocation. Although 
Table 1. Biomarkers of sepsis analyzed in immunocompetent patients and/or patients with HIV/AIDS. Diagnostic or prognostic value of biomarkers of sepsis

\begin{tabular}{|c|c|c|c|c|}
\hline $\begin{array}{l}\text { Sepsis } \\
\text { biomarker }\end{array}$ & $\begin{array}{c}\text { Analyzed } \\
\text { in immunocompetent } \\
\text { patients }\end{array}$ & $\begin{array}{l}\text { Analyzed in patients } \\
\text { with HIV/AIDS }\end{array}$ & Diagnostic value & Prognostic value \\
\hline PCT & Yes & Yes & Yes & Yes in immunocompetent patients \\
\hline IL-6 & Yes & Yes & Yes & Yes \\
\hline SUPAR & Yes & Not as a sepsis biomarker & No & Yes \\
\hline proADM & Yes & Not as a sepsis biomarker & Yes & Yes \\
\hline STREM-1 & Yes & Not as a sepsis biomarker & Yes & No \\
\hline Gas6 & Yes & Not as a sepsis biomarker & No & Yes \\
\hline sCD14-ST & Yes & Not as a sepsis biomarker & Yes & Yes \\
\hline PSP & Yes & No & No & Yes \\
\hline HBP & Yes & No & No & Yes \\
\hline miRNA & Yes & Not as a sepsis biomarker & Yes & Yes \\
\hline
\end{tabular}

analyzed carefully in many studies and considered by many authors a promising biomarker of sepsis, it has not been yet analyzed in patients with HIV/AIDS in that context.

\section{Pancreatic stone protein / regenerating protein (PSP/reg)}

$\mathrm{PSP} /$ reg is secreted by pancreatic acinar cells into pancreatic juice and by subsets of intestinal and gastric cells. $\mathrm{PSP} / \mathrm{reg}$ is considered to be a new protein present in acute phase of inflammation. Some studies showed that when measured within 24 hours of ICU admission, it may predict in-hospital mortality in patients with sepsis. In another study, PSP/reg, PCT, and high sensitivity-CRP (hs-CRP) were independent risk factors for prognosis of sepsis in pediatric patients. This analysis was not conducted on a cohort of adult patients, nor on a cohort of patients with HIV/AIDS [51].

\section{Heparin-binding protein (HBP)}

HBP is secreted from activated neutrophils after a contact with endothelium and from azurophil granules after internalization of bacteria by monocytes. In 2015, Linder et al. suggested that HBP could be used as a predictor of organ dysfunction during infection in patients admitted to emergency departments [52].

In the next years, in many trials, significance of HBP as a biomarker of sepsis and inflammation confirmed effectiveness of HBP $[53,54]$. However, we did not find any papers analyzing $\mathrm{HBP}$ as a sepsis biomarker in HIV-infected patients.

\section{Discussion}

HIV infection leads to disorders of the humoral and cellular immune response. Initially, the number of CD4+ T lymphocytes is reduced, but with time it grows, only to be fol- lowed by a decrease in numbers again. In subsequent years, the function of CD4+ T lymphocytes is irreversibly damaged, leading to changes in the production of various cytokines. These changes in the immune response have led to a hypothesis that HIV might lead to further changes in the host response in sepsis. But in the era of treating all patients with $\mathrm{HIV}$, independent of CD4+ cell count, changes in the immune system in patients on antiretroviral treatment (ART) do not differ much from that in immunocompetent individuals. Therefore, the focus should be on prompt diagnosis and treatment of patients with AIDS who are not yet on ART.

About $12-31 \%$ of admissions of patients with HIV to ICU is due to sepsis [55]. In a retrospective study by VidalCortes et al., a total of $104 \mathrm{HIV}$-infected patients were admitted to ICU, in which the most frequent sites of infection were the lungs (more than $65.0 \%$ ), followed by central nervous system (16.4\%), urinary tract infection, and infective endocarditis, both occurring in $4.9 \%$ of patients. ICU hospital mortality rate was $41.9 \%$. The most common pathogens included Streptococcus pneumoniae (28.8\%), Pneumocystis jirovecii (13.6\%), Toxoplasma gondii (8.5\%), Escherichia coli, and Haemophilus influenzae (both, 5.1\%) [56].

In previous studies, patients with HIV/AIDS and sepsis had a higher mortality than those with sepsis but without HIV [57, 58]. In 2013, Silva et al. in a prospective study showed that although the severity of sepsis and prognostic scores were similar between patients with and without HIV, the mortality was higher for HIV patients. Mycobacterial and fungal etiologies were the most frequent causes of infections, leading to pulmonary and abdominal diseases [55]. A different result was presented by Wiewel et al. in 2016, who in a prospective study of 1,889 patients with sepsis (32 patients with HIV), measured 14 biomarkers (INF- $\alpha$, IL-1 $\beta$, IFN- $\gamma$, IL-6, IL-8, IL-10, IL-13, ICAM, soluble E-selectin, angiopoetin-1 and 2, protein $\mathrm{C}$, and antithrombin), which play an important role in sepsis, and showed that the concentration level of these biomarkers were similar in patients 
with and without HIV. Moreover, the disease severity and outcome was similar for patients with and without HIV. The only statistically significant difference was that patients with HIV had a higher prevalence of pneumonia [59].

Differences in results might be that patients' selection and setting of the study differed. In a paper by Wiewel et al., $70.7 \%(n=29)$ of patients were already on treatment, and $47.4 \%(n=18)$ had an undetectable viral load [59]. Patients with an advanced HIV disease (CD4+ cell count $<200$ cells/ $\mu \mathrm{l}$ or AIDS-defining illness) are at higher risk of developing opportunistic infections (OIs), and with unknown HIV status, the detection and treatment might be delayed.

Even though in the era of ART, main causes of death of HIV-positive patients are non-AIDS-related events, and about $49 \%$ of newly diagnosed HIV cases in Europe are late presenters (CD4+ cell count $<350$ or with an AIDS-related event, regardless of CD4+ cell count) [60]. Therefore, these cases constitute a group of patients that need prompt diagnosis and treatment.

Early diagnosis of OIs could prevent the development of sepsis. Markers that might be useful in differentiating community-acquired pneumonia (CAP) from tuberculosis are $\mathrm{C}$-reactive protein (CRP), procalcitonin (PCT), and soluble form of urokinase-type plasminogen activator receptor (suPAR).

Even though CRP is not a sepsis biomarker itself, it is an important marker of inflammation, and in HIV/AIDS patients, it might be important for diagnosis of TB and CAP. In a meta-analysis evaluating diagnostic accuracy of CRP (10 mg/l cut-off point) among patients with Mycobacterium tuberculosis (MTB), it was shown that CRP could be used to screen for tuberculosis (TB) in patients with HIV/AIDS [61]. Similar results were also showed by Bedell et al., where elevated CRP was associated with confirmed or probable TB infection in a group of $452 \mathrm{HIV}$-infected adults from Malawi [62].

To our knowledge, many of the biomarkers of sepsis discussed in this article have not yet been analyzed in patients with HIV/AIDS. The value of the biomarkers discussed in this paper is summarized in Table 1. Combining at least two different biomarkers has the best potential to bring high sensitivity and specificity of diagnosis of sepsis.

\section{Conclusions}

So far, there is not a one biomarker used as a golden standard in the diagnosis of sepsis. Using two or more biomarkers might increase the chance of early detection of sepsis, and thus be beneficial for patients. To our knowledge, many biomarkers were not yet analyzed in a population of patients with AIDS. Further research is needed in finding biomarkers diagnosing sepsis in HIV/AIDS patients.

\section{Conflict of interest}

The authors declare no conflict of interest.

\section{References}

1. Singer M, Deutschman CS, Seymour CW, et al. The third international consensus definitions for sepsis and septic shock (Sepsis-3). JAMA 2016; 315: 801-810.

2. Morrow KN, Coopersmith CM, Ford ML. IL-17, IL-27 and IL-33: a novel axis linked to immunological dysfunction during sepsis. Front Immunol 2019; 10: 1982.

3. Angus DC. The lingering consequences of sepsis: a hidden public health disaster? JAMA 2010; 304: 1833-1834.

4. Biomarkers Definitions Working Group. Biomarkers and surrogate endpoints: preferred definitions and conceptual framework. Clin Pharmacol Ther 2001; 69: 89-95.

5. Pierrakos C, Vincent JL. Sepsis biomarkers: a review. Crit Care 2010; 14: R15.

6. Chirouze C, Schuhmacher H. Low serum PCT level accurately predicts the absence of bacteriemia in adult patients with acute fever. Clin Inf Dis 2002; 35: 156-161.

7. Jones AE, Fiechtl JF, Brown MD, et al. Procalcitonin test in the diagnosis of bacteremia: a meta-analysis. Ann Emerg Med 2007; 50: 34-41.

8. Dolin HH, Papadimos TJ, Stepkowski S, et al. A novel comibination of biomarkes to herald the onset of sepsis prior to the manifestation of symptoms. Shock 2018; 49: 364-370.

9. Zhang T, Wang Y, Yang Q, et al. Procalcitonin-guided antibiotic therapy in critically ill adults: a meta-analysis. BMC Infect Dis 2017; 17: 514 .

10. Jensen JU, Heslet L, Jensen TH, et al. Procalcitonin increase in early identification of critically ill patients at high risk of mortality. Crit Care Med 2006; 34: 2596-2602.

11. Bele N, Darmon M, Coquet I, et al. Diagnostic accuracy of procalcitonin in critically ill immunocompromised patients. BMC Infect Dis 2011; 11: 224.

12. Kang YA, Kwon SY, Yoon HI, et al. Role of C-reactive protein and procalcitonin in differentiation of tuberculosis from bacterial community acquired pneumonia. Korean J Intern Med 2009; 24: 337-342.

13. Schleicher GK, Herbert V, Brink A, et al. Procalcitonin and C-reactve protein level in HIV-positive subjects with tuberculosis and pneumonia. Eur Respir J 2005; 25: 688-692.

14. Nyamande K, Lalloo UG. Serum procalcitonin distinguishes CAP due to bacteria, Mycobacterium tuberculosis and PJP. Int J Tuberc Lung Dis 2006; 10: 510-515.

15. Janssen S, Schutz C, Ward A, et al. Mortality in severe human immunodeficiency virus-tuberculosis associates with innate immune activation and dysfunction of monocytes. Clin Infect Dis 2017; 65: 73-82.

16. Bokun LV, Huang J, Yuan H, et al. Tumor necrosis factor- $\alpha$ as a diagnostic marker for neonatal sepsis: a meta-analysis. Sci World J 2014; 2014: 471463.

17. Wolf J, Rose-John S, Garbers C. Interleukin-6 and its receptors: a highly regulated and dynamic system. Cytokine 2014; 70: 11-20.

18. Wong HR, Cvijanovich NZ, Hall M, et al. Interleukin-27 is a novel candidate diagnostic biomarker for bacterial infection in critically ill children. Crit Care 2012; 16: R213.

19. Wong HR. Performance of IL-27 as a sepsis diagnostic biomarker in the critically ill adults. J Crit Care 2014; 29: 718-722.

20. Amancio RT, Japiassu AM, Gomes RN, et al. The innate immune response in HIV/AIDS septic shock patients: a comparative study. PLoS One 2013; 8: e68730.

21. Taniguchi T, Koido Y, Aiboshi J, et al. Change in the ratio of interleukin- 6 to interleukin-10 predicts a poor outcome in patients with systemic inflammatory response syndrome. Crit Care Med 1999; 27: 1262-1264.

22. Sun J, Su J, Xie Y, et al. Plasma IL-6/IL-10 ratio and IL-8, LDH, and HBDH level predict the severity and the risk of death in AIDS patients with pneumocystis pneumonia. J Immunol Res 2016; 2016: 1583951. 
23. Sandquist M, Wong HR. Biomarkers of sepsis and their potential value in diagnosis, prognosis and treatment. Exp Rev Clin Immunol 2014; 10: 1349-1356.

24. Ni W, Han Y, Zhao J, et al. Serum soluble urokinase-type plasminogen activator receptor as a biological marker of bacterial infection in adults: a systematic review and meta-analysis. Sci Rep 2016; 6: 39481.

25. Suberviola B, Castellanos-Ortega A, Ruiz Ruiz A, et al. Hospital mortality prognostication in sepsis using the new biomarkers suPAR and proADM in a single determination on ICU admission. Intensive Care Med 2013; 39: 1945-1952.

26. Henriquez-Camacho C, Losa J. Biomarkers for sepsis. Biomed Res Int 2014; 2014: 547818.

27. Sidenius N, Sier CF, Ullum H, et al. Serum level of soluble urokinase-type plasminogen activator receptor is a strong and independent predictor of survival in human immunodeficiency virus infection. Blood 2000; 96: 4091-4095.

28. Kirkegaard-Klitbo DM, Langkilde A, Mejer N, et al. Soluble urokinase plasminogen activator receptor is a predictor of incident non-AIDS comorbidity and all-cause mortality in human immunodeficiency virus type 1 infection. J Infect Dis 2017; 216: 819-823.

29. Rasmussen LJ, Knudsen A, Katzenstein TL, et al. Soluble urokinase plasminogen activator receptor (suPAR) is a novel, independent predictive marker of myocardial infarction in HIV-1-infected patients: a nested case-control study. HIV Med 2016; 17: 350-357.

30. Akpinar S, Rollas K, Alagoz A, et al. Performance evaluation of MRproadrenomedullin and other scoring systems in severe sepsis with pneumonia. J Thorac Dis 2014; 6: 921-929.

31. Spoto S, Cella E, de Cesaris M, et al. Procalcitonin and Mr-proadrenomedullin combination with SOFA and qSOFA scores for sepsis diagnosis and prognosis: a diagnostic algorithm. Shock 2018; 50: 44-52.

32. Angeletti A, Battistoni F, Fioravanti M, et al. Procalcitonin and midregional pro-adrenomedullin test combination in sepsis diagnosis. Clin Chem Lab Med 2013; 5: 1059-1067.

33. Albrich WC, Madhi SA, Adrian PV, et al. Pneumococcal colonisation density: a new marker for disease severity in HIV-infected adults with pneumonia. BMJ Open 2014; 4: e005953.

34. Bouchon A, Dietrich J, Colonna M. Cutting edge: inflammatory responses can be triggered by TREM-1, a novel receptor expressed on neutrophils and monocytes. J Immunol 2000; 164: 4991-4995.

35. Gibot S, Cravoisy A, Levy B, et al. Soluble triggering receptor expressed on myeloid cells and the diagnosis of pneumonia. N Engl J Med 2004; 350: 451-458.

36. Su L, Xie L, Liu D. Urine sTREM-1 may be a valuable biomarker in diagnosis and prognosis of sepsis-associated acute kidney injury. Crit Care 2015; 19: 281.

37. Wu Y, Wang F, Fan X, et al. Accuracy of plasma sTREM-1 for sepsis diagnosis in systemic inflammatory patients: a systematic review and meta-analysis. Crit Care 2012; 16: R229.

38. Aksaray S, Alagoz P, Inan A, et al. Diagnostic value of sTREM-1 and procalcitonin levels in the early diagnosis of sepsis. North Clin Istanb 2017; 3: 175-182.

39. Hyun J, McMahon RS, Lang AL, et al. HIV and HCV augments inflammatory responses through increased TREM-1 expression and signaling in Kupffer and Myeloid cells. PLoS Pathog 2019; 15: e1007883.

40. Hsiao FC, Lin YF, Hsieh PS, et al. Circulating growth arrest-specific 6 protein is associated with adiposity, systemic inflammation, and insulin resistance among overweight and obese adolescents. J Clin Endocrinol Metab 2013; 98: 267-274.

41. Borgel D, Clauser S, Bornstain C, et al. Elevated growth-arrest-specific protein 6 plasma levels in patients with severe sepsis. Crit Care Med 2006; 34: 219-222.

42. Stalder G, Que YA, Calzavarini S, et al. Study of early elevated Gas6 plasma level as a predictot of mortality in a prospective cohort of patients with sepsis. PLoS One 2016; 11: e0163542.
43. Chua BA, Ngo JA, Situ K, Morizono K. Roles of phosphatidylserine exposed on the viral envelope and cell membrane in HIV-1 replication. Cell Commun Signal 2019; 17: 132.

44. Benz F, Roy S, Trautwein C, Roderburg C, Luedde T. Circulating microRNAs as biomarkers for sepsis. Int J Mol Sci 2016; 17: 78.

45. Zhang W, Jia J, Liu Z, Si D, Ma L, Zhang G. Circulating microRNAs as biomarkers for sepsis secondary to pneumonia diagnosed via sepsis 3.0. BMC Pulm Med 2019; 19: 93.

46. Balasubramaniam M, Pandhare J, Dash C. Are microRNAs important players in HIV-1 infection? An update. Viruses 2018; 10: 110.

47. Jin C, Cheng L, Hoxtermann S, et al. MicroRNA-155 Is a Biomarker of T-cell Activation and Immune Dysfunction in HIV-1-infected Patients. HIV Med 2017; 18: 354-362.

48. Mussap M, Noto A, Fravega M, Fanos V. Soluble CD14 subtype presepsin (sCD14-ST) and lipopolysaccharide binding protein (LBP) in neonatal sepsis: new clinical and analytical perspectives for two old biomarkers. J Matern Fetal Neonatal Med 2011; 24: 12-14.

49. Wu J, Hu L, Zhang G, Wu F, He T. Accuracy of presepsin in sepsis diagnosis: a systematic review and meta-analysis. PLoS One 2015; 10: $\mathrm{e} 0133057$.

50. Tong X, Cao Y, Yu M, Han C. Presepsin as a diagnostic marker for sepsis: evidence from a bivariate meta-analysis. Ther Clin Risk Manag 2015; 11: 1027-1033.

51. Wu Q, Nie Y, Wu FX, Zou XL, Chen FY. Prognostic value of highsensitivity c-reactive protein, procalcitonin and pancreatic stone protein in pediatric sepsis. Med Sci Monit 2017; 23: 1533-1539.

52. Linder A, Arnold R, Boyd JH, et al. Heparin-binding protein measurement improves the prediction of severe infection with organ dysfunction in the emergency department. Crit Care Med 2015; 43 : 2378-2386.

53. Chen S, Zhang C, Hong G, et al. Meta-analysis of the diagnostic efficacy of heparin binding protein in adult sepsis. Zhonghua Wei Zhong Bing Ji Jiu Yi Xue 2019; 31: 1330-1334.

54. Holub M, Dzupova O, Ruzkova M, et al. Selected biomarkers correlate with the origin and severity of sepsis. Mediators Inflamm 2018; 2018: 7028267.

55. Silva JM Jr, De Sousa dos Santos S. Sepsis in AIDS patients: clinical, etiological and inflammatory characteristics. J Int AIDS Soc 2013; 16: 17344

56. Vidal-Cortés P, Lameiro-Flores P, Mourelo-Fariña M, et al. Sepsis in HIV patients admitted to the ICU. Critical Care 2013; 17: P513.

57. Mrus JM, Braun LA, Yi MS, Linde-Zwirble WT, Johnston JA. Impact of HIV/AIDS on care and outcomes of severe sepsis. Critical Care 2005; 9: R623-R630.

58. Cribbs S. Characteristics and outcomes of HIV-infected patients with severe sepsis: continued risk in the post-highly active antiretroviral therapy era. Crit Care Med 2015; 43: 1638-1645.

59. Wiewel MA, Huson MA, van Vught LA, et al. Impact of HIV infection on the presentation, outcome and host response in patients admitted to the intensive care unit with sepsis, a case control study. Crit Care 2016; 20: 322.

60. ECDC, Infographic: HIV late diagnosis - 2018 data. Available at: https://www.ecdc.europa.eu/en/publications-data/infographic-hivlate-diagnosis-2018-data.

61. Yoon C, Chaisson LH, Patel SM, et al. Diagnostic accuracy of C-reactive protein for active pulmonary tuberculosis: a meta-analysis. Int J Tuberc Lung Dis 2017; 21: 1013-1019.

62. Bedell RA, van Lettow M, Meaney C, et al. Predictive value of C-reactive protein for tuberculosis, bloodstream infection or death among HIV-infected individuals with chronic, non-specific symptoms and negative sputum smear microscopy. Trop Med Int Health 2018; 23: 254-262. 